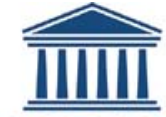 \\ UNIVERSITEIT \\ GENT
}

biblio.ugent.be

The UGent Institutional Repository is the electronic archiving and dissemination platform for all UGent research publications. Ghent University has implemented a mandate stipulating that all academic publications of UGent researchers should be deposited and archived in this repository. Except for items where current copyright restrictions apply, these papers are available in Open Access.

This item is the archived peer-reviewed author-version of:

Title: Thymidine monophosphate kinase from Mycobacterium tuberculosis

Drug design and identification of potent leads

Authors: S. Van Calenbergh, S. Pochet and H. Munier-Lehmann

In: Curr. Top. Med. Chem., 2012, 12, 694-705 


\section{Thymidine monophosphate kinase from Mycobacterium tuberculosis Drug design and identification of potent leads}

S. Van Calenbergh ${ }^{1}$, S. Pochet ${ }^{2}$ and H. Munier-Lehmann ${ }^{2, *}$

\footnotetext{
${ }^{1}$ Laboratory for Medicinal Chemistry (FFW), Ghent University, B-9000 Gent, Belgium; ${ }^{2}$ Institut Pasteur, Unité de Chimie Organique, CNRS URA2128, Paris 15, France
}

*Address correspondence to this author at the Institut Pasteur, Unité de Chimie Organique, CNRS URA2128, 28 Rue du Dr Roux, 75724 Paris cedex 15, France; Tel: (0033) 1456883 81; Fax: (0033) 145688404 ; Email: helene.munier-lehmann@pasteur.fr 


\section{ABSTRACT}

Antiviral chemotherapy often relies on nucleoside analogues, which, once phophorylated by intracellular kinases, target viral polymerases and preclude DNA synthesis. In contrast, nucleoside analogues are much less explored as antibacterial drugs. TMPKmt, which is essential to DNA replication, was selected as a promising target for inhibitor design. This review will describe how stepwise modifications of the enzyme's substrate, guided by the feedback of the enzyme assays as well as crystallographic information, proved a valuable approach to produce potent TMPKmt inhibitors, some of which were also capable to inhibit bacterial growth. Perhaps more importantly, some of the reported thymidine analogues also represent valuable tools to better understand the structure and the mechanism of this intriguing enzyme.

\section{INTRODUCTION}

Once nearly vanquished by antibiotics, at least in the developed world, tuberculosis (TB) resurged in the late 1980's and now kills more than 2 million people a year - second only to AIDS among infectious diseases. Thirty million people are at risk of dying from TB in the next 10 years, most of them living in Africa [1]. Mycobacterium tuberculosis, the causative agent of TB, is a slow-growing bacillus, primarily transmitted via the respiratory route, mostly causing pulmonary TB. Estimates are that one third of the world's population is infected with this tenacious and remarkably successful pathogen, but infection usually does not lead to the active disease. Reactivation of this latent infection can be caused by immunodeficiency, HIV infection, use of corticosteroids, aging, alcohol and drug abuse. In HIV infected patients, TB has become the main cause of death. Next to HIV-infection, demographic factors, socio-economic trends and the neglected TB control in many countries caused this vast epidemic.

The increasing problem of MDR-TB [2] and the lack of an effective vaccine [3] make the development of new drugs, that not only act against drug-resistant TB, but also shorten the lengthy therapy, a global emergency [4, 5]. In developing new TB drugs, it is crucial to determine which targets in the tubercle bacillus are drugable. The complete genome sequence of the $M$. tuberculosis H37Rv strain has been recently determined [6] and analyzed. This milestone, in concert with mycobacterial genetic tools, has greatly facilitated the identification of targets involved in bacterial growth, metabolism and viability, whose inactivation will lead to bacterial death or inability to persist $[7,8]$. 
In this review we will focus on a housekeeping enzyme, namely thymidine monophosphate kinase (TMPK) from M. tuberculosis (TMPKmt). This enzyme is part of the nucleotide biosynthesis, which is a cornerstone target for antiviral and cancer chemotherapy. Different approaches for the development of inhibitors of TMPKmt as potential leads for new antimycobacterial drugs will be described.

\section{TMPKmt}

\section{An attractive target}

Thymidine monophosphate kinase (TMPK, also called ATP:dTMP phosphotransferase or thymidylate kinase) belongs to the nucleoside monophosphate kinases (NMPKs) family [9]. It catalyses the reversible phosphorylation of thymidine monophosphate (dTMP) to thymidine diphosphate (dTDP) utilizing ATP as its preferred phosphoryl donor. Situated at the junction of the de novo and salvage pathways for the synthesis of thymidine triphosphate (dTTP), TMPK is the last specific enzyme in these pathways. This enzyme is indispensable for growth and survival and, therefore, represents a promising target for developing new TB drugs. With experiments using a cdc8 mutant of Saccharomyces cerevisiae, deficient in TMPK, Jong et al. proved that yeast TMPK (TMPKy) is essential for DNA replication and thus for cellular growth [10].

Although the global folding of TMPKmt [11] is similar to that of other TMPKs, the configuration of its active site is unique. Moreover, the enzyme possesses peculiar properties such as AZT-MP being a competitive inhibitor [12], suggesting that selective blocking of the bacterial DNA synthesis should be feasible. The latter could also be achieved upon incorporation of modified nucleoside triphosphates acting as chain terminators of forming DNA. As phosphorylated derivatives are not membrane permeable, nucleotides should preferably be delivered as their nucleoside precusors, which are then converted to their phosphorylated counterparts by intracellular kinases. This is the case for AZT or other nucleoside analogs used in the HIV therapy. The first phosphorylation step is achieved by thymidine kinase (TK) which phosphorylates thymidine (dT) to dTMP. For mycobacteria, neither a gene coding for a TK has been identified in the genome of different mycobacteria species [6, 13-15] nor any TK activity in crude extracts [16]. However, dT as well as other 5'-modified analogs (5'-azido- or 5'amino-5'-deoxy-thymidine) were found to be competitive inhibitors of TMPKmt with affinities in the same range as for dTMP [17], indicating that unphosphorylated nucleoside analogs could act directly on the enzyme without any metabolic processing, thereby making TMPKmt an attractive target for antituberculosis drugs. 


\section{Catalytic properties of TMPKmt}

TMPKmt has a broad specificity for nucleoside triphosphates as phosphate donors, and as expected a very narrow one for nucleoside monophosphates as phosphate acceptors [12]. This is a general feature of TMPKs and other members of the NMPK family [9]. However, TMPK from Plasmodium falciparum has been recently described as a notable exception [18]: this enzyme is able to phosphorylate not only pyrimidine (dTMP, dUMP) but also purine (dGMP, dIMP and GMP) nucleotides. The catalytic activity of TMPKmt is low [12] compared to TMPK from Escherichia coli (TMPKec) and TMPKy, and TMPKmt shows a high resistance to thermal denaturation. On the other hand, the $K_{m}$ values for dTMP and ATP lie in the same order of magnitude for TMPKmt, human TMPK (TMPKh), TMPKec and TMPKy (see Table 1).

\begin{tabular}{lcccc}
\hline TMPK & $\begin{array}{c}\mathbf{K}_{\mathrm{m}}{ }^{\text {ATP }} \\
(\mathrm{mM})\end{array}$ & $\begin{array}{c}\mathbf{K}_{\mathrm{m}}{ }^{{ }^{\mathrm{TTMP}}} \\
(\mu \mathrm{M})\end{array}$ & $\begin{array}{c}\mathbf{V}_{\mathbf{m}}{ }^{\text {ATP,dTMP }} \\
(\mu \text { mole/min } / \mathrm{mg} \\
\text { of protein })\end{array}$ & $\begin{array}{c}\mathbf{k}_{\text {cat }} \\
\left(\mathrm{s}^{-1}\right)\end{array}$ \\
\hline M. tuberculosis & 0.1 & 4.5 & 13 & 4.5 \\
E. coli & 0.04 & 15 & 50 & 10.5 \\
Yeast & 0.19 & 9 & 84 & 35 \\
Human & 0.03 & 5 & 3 & 1.6 \\
\hline
\end{tabular}

Table 1. Kinetic parameters for natural substrates of TMPKy [19], TMPKmt, TMPKec, and TMPKh [12].

\section{X-ray structures of TMPKmt}

TMPKmt has been crystallized for the first time as a complex with its natural substrate dTMP [11]. It is a homodimer, each monomer of 214 residues being composed of nine $\alpha$-helices surrounding a five-stranded $\beta$ sheet core. This global folding is similar to that of other TMPKs, despite the low similarity of their primary sequences: $26 \%, 25 \%$ and $22 \%$ sequence identity over about 200 aligned residues with TMPKec, TMPKy and TMPKh respectively.

Three regions contain the essential residues for the catalytic activity of TMPKmt (Fig. 1):

- the P-loop motif [20] (residues 7-14, $\mathrm{GlyX}_{1} \mathrm{X}_{2} \mathrm{X}_{3} \mathrm{X}_{4}$ GlyLys $\mathrm{X}_{5}$ ) controls the positioning of the phosphoryl groups of the phosphate donor. It binds and positions the $\alpha$ - and $\beta$-phosphoryl groups of ATP through interactions between amide backbone hydrogens and phosphate oxygen atoms. Unique to TMPKs is 
an interaction between the P-loop and the phosphoryl acceptor dTMP. This interaction is achieved through a hydrogen bond between the 3'-hydroxyl group of dTMP and a carboxylic acid residue at position $\mathrm{X}_{2}$ of the Ploop motif (D9 in TMPKmt). The exact function of this acidic amino acid (D14 in TMPKy, D15 in TMPKh, and E12 in TMPKec) which played a key role in initial efforts to design inhibitors, is still unclear but it seems to be crucial for the catalytic mechanism of TMPK as any mutation of this residue in either TMPKy or TMPKh abolishes the phosphoryl transfer activity [21].

- the $\mathrm{DR}(\mathrm{Y} / \mathrm{H} / \mathrm{F})$ motif is a loop containing a strictly conserved arginine residue, acting as a clip which favorably orients the phosphoryl donor and acceptor via an interaction with the phosphate group of dTMP and the $\gamma$-phosphate of ATP.

- the LID region (residues 147-159) as in all NMPKs is a flexible stretch that undergoes substantial conformational changes: a transition from a coil to a helical conformation in TMPKy when ATP becomes bound [19]. In most NMPKs, this closure of the LID region brings catalytic arginines to the reaction centre. Eukaryotic TMPKs deviate from this paradigm in that they lack positively charged residues in the LID region. This features led to a categorization of TMPKs into two types [22]. Type I TMPKs (e.g. TMPKy and TMPKh) have, in addition to the invariant lysine (K19 in TMPKh), a basic residue (arginine) around position 16 in their P-loop sequence that can interact with the $\gamma$-phosphate of ATP and lack such a positively charged residue in the LID region. Type II TMPKs (e.g. TMPKec) have a glycine residue at that place in the P-loop and one additional basic residue in the LID region that interacts with ATP (R153 in TMPKec). TMPKmt displays a configuration essentially representative of type II TMPKs, the LID region comprising a series of basic residues (R149, R151, R153 and R156) and the P-loop having the usual glycine residue (G10) at position $\mathrm{X}_{3}$. However, a peculiar additional basic residue, R14, is found at position $\mathrm{X}_{5}$ in the P-loop, partly reminiscent of type I TMPKs, but unique among all TMPKs. Another main difference concerns the LID closure, which is, for TMPKmt, not due to ATP binding but to the binding of $\mathrm{a} \mathrm{Mg}^{2+}$ ion in the dTMP-binding site [23]. 


\section{- P-loop -}

mt - - - - - MLIAIEGVDGAGKRTLVEKLSGAFRAAG - RSVATLAFPRYGQSVAADIAAEALHGEH SC MMG-RGKLILIEGLDRTGKTTTQCNILYKKLQP - - - NCKLLKFPER-STRIGGLINEYLTDD - $\mathrm{h}$ MAARRGALIVLEGVDRAGKSTQSRKLVEALCAAGHR-AELLRFPER-STEIGKLLSSYLQKK - ec - - MRSKYIVIEGLEGAGKTTARNVVVETLEQLGIRDMVFTREPG - - GTQLAEKLRSLLLDIKS

$\star * \star$

mt -GDLASSVYAMATLFALDRAGAVHTIQGLCRGY - DVVILDRYVASNAAYSAARLHENAAGKAA

Sc - -SFQLSDQAIHLLFSANRWEIVDKIKKDLLEG - KNIVMDRYVYSGVAYSAA - - KGTNGMDL

$\mathrm{h}$ - - SD - VEDHSVHLLFSANRWEQVPLIKEKLSQG -VTLVVDRYAFSGVAFTGA - - K - EN - FSL

ec VGDEVITDKAEVLMFYAARVQLVETVIKPALANGTWVIGDRHDLSTQAYQGG - - GRG - - - IDQ $\alpha 5$

mt ELQQRTGAVYAELAAQGW - - - GGRWLVVGAD - VDPGRLAATLAPPDVPS - - - - - - - - - - - - -

$$
\alpha 7
$$$$
-\beta 5-
$$
$\alpha 8$

Fig. (1). Structure-based sequence alignment of TMPKmt (mt), TMPKy (sc), TMPKh (h) and TMPKec (ec). The secondary structural elements of TMPKy as well as the P-loop and the LID domain are shown under and above the alignment, respectively. The LID domains of TMPKy and TMPKh were not aligned to those of TMPKmt and TMPKec as their backbone conformations differ greatly and do not superimpose. The $D R(Y / H / F)$ motif is marked by asterisks. Underlined and bold residues are mentioned in the text. 
Like other NMPKs, TMPKmt undergoes large conformational changes upon substrate binding. One can distinguish between the open conformation, observed without substrate, the partially closed conformation, observed with a single substrate, and the fully closed and active conformation, observed in the presence of both substrates (dTMP and ATP). In the 3D-structure described by Li de la Sierra et al. [11], TMPKmt is in the fully closed conformation despite the fact that the enzyme was cocrystallized as a complex with dTMP and $\mathrm{SO}_{4}{ }^{2-}$, in the absence of ATP. Indeed, the structural characteristics observed around the ATP binding site are similar to those observed in crystal structures of TMPKy and TMPKec where ATP was fixed. The structural changes of TMPKmt occurring upon binding of the substrates and subsequent catalysis were elegantly investigated by X-ray experiments [23]. They were aligned with an induced-fit mechanism already suggested for several NMPKs $[24,25]$ and with the idea of an ordered bi-bi-catalytic mechanism, in contrast to the random bi-bi-mechanism generally proposed for these enzymes [26]. The possibility of a mechanism involving ATP binding first cannot be completely excluded from this study. However, it has been shown that dTMP exhibits an affinity $\left(K_{m}=4.5 \mu M\right)$ for the enzyme more than 20 times stronger than ATP $\left(K_{m}=100 \mu M\right)$, suggesting that the pathway involving dTMP binding first is largely favored when both substrates are present in the same concentration [12]. Closure of the LID region upon ATP binding would sterically restrict the access of dTMP to its binding site or require a secondary channel through which dTMP may enter the active site.

Another unique feature of TMPKmt is that the magnesium ion lies in the dTMP-binding site. Instead of a NTPbound $\mathrm{Mg}^{2+}$ as typically found in NMPKs, it is coordinated by an oxygen atom from the phosphoryl group of dTMP, four water molecules and the carboxylate oxygen atoms of D9 and E166. This cation is supposed to neutralize the electrostatic repulsion between the anionic substrates and to act as a clamp for the proper alignment of the phosphoryl acceptor and donor.

\section{The dTMP-binding site of TMPKmt}

Examination of the X-ray structure of TMPKmt [11] reveals that the main binding forces between dTMP and the enzyme are the following (Fig. 2): (i) a stacking interaction between the pyrimidine ring and F70; (ii) a hydrogen bond between the $\mathrm{O}^{4}$ of thymine and the R74 side-chain, which results in a preference for thymine over cytosine; (iii) a hydrogen bond between $\mathrm{N} 100$ and the $\mathrm{N}^{3}$ of the thymine ring; (iv) a hydrogen bond between the 3'-hydroxyl of dTMP and the terminal carboxyl of D9, that in turn interacts with the magnesium ion responsible for positioning the phosphate oxygen of dTMP. The 3'-OH group actually makes three polar contacts: one with a water molecule involved in the $\mathrm{Mg}^{2+}$-coordination and two others with $\mathrm{D9}$; (v) hydrogen bonds between the 5'- 
O-phosphoryl and Y39, F36 and an ionic interaction with R95 and $\mathrm{Mg}^{2+}$. Y39 makes two polar contacts with dTMP: one with the phosphate oxygen atom and the other one with the oxygen atom at the 5'-position. An arginine residue replaces this tyrosine in TMPKh; (vi) the presence of $\mathrm{Y} 103$ close to the 2'-position is believed to render the enzyme catalytically selective for 2'-deoxyribo-nucleotides versus ribonucleotides.

These differences are potentially interesting starting points for finding inhibitors that exert selectivity for TMPKmt over TMPKh.
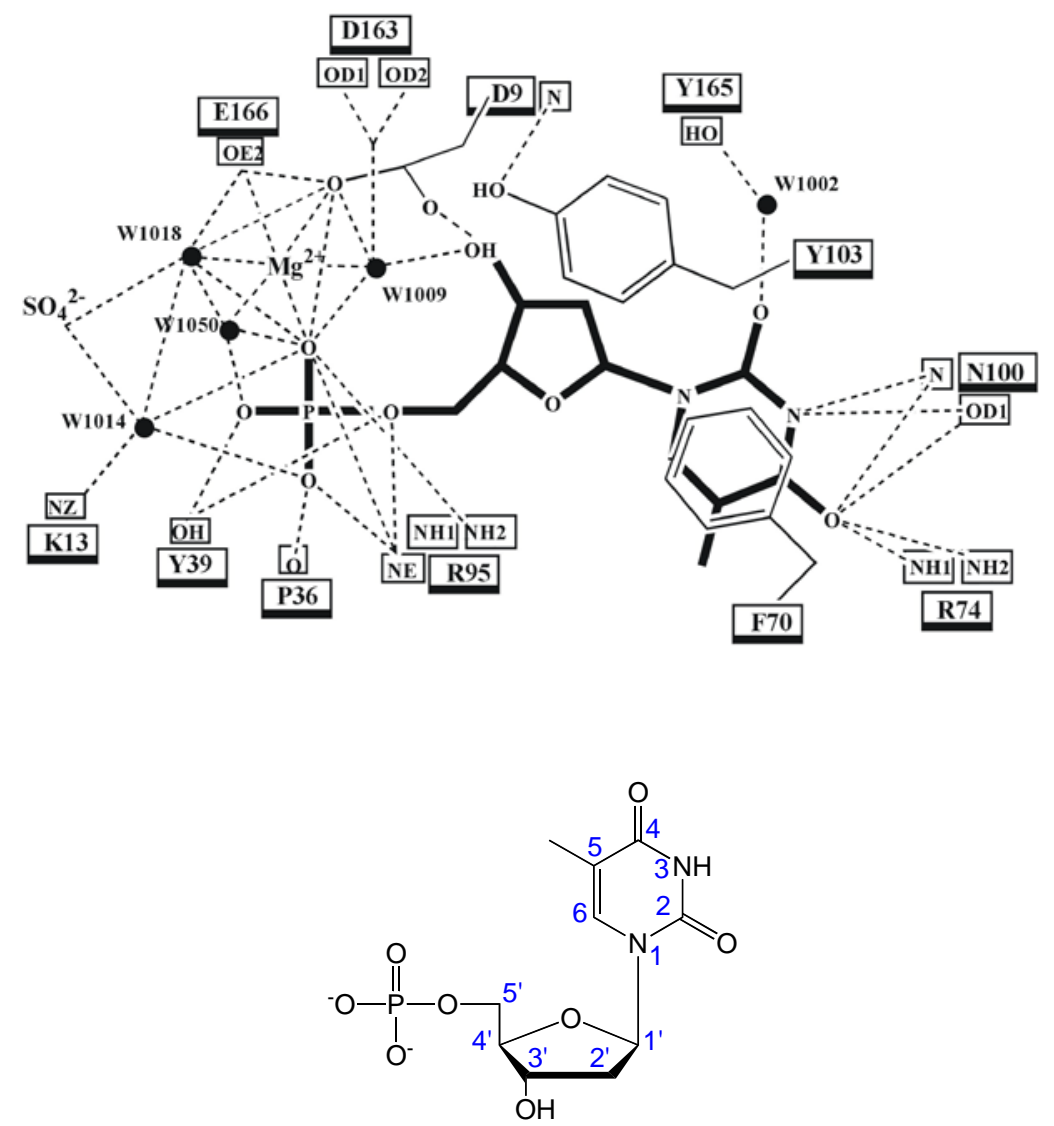

Fig. (2). Top: Schematic drawing of the dTMP (bulk lines) binding site of TMPKmt [11]. Bottom: Structure and atom numbering of dTMP.

\section{DESIGN OF INHIBITORS OF TMPKMT}

\section{Initial structure-activity relationship}

The first modified dTMP analogs to have been reported are listed in Table 2 [12]. 


\begin{tabular}{|c|c|c|c|}
\hline NMP & $\mathrm{K}_{\mathrm{m}}^{\mathrm{app}}(\mu \mathrm{M})$ & $\begin{array}{c}\mathrm{V}_{\mathrm{m}}(\mu \mathrm{mole} / \mathrm{min} / \mathrm{mg} \\
\text { of protein) }\end{array}$ & $\mathrm{K}_{\mathrm{i}}(\mu \mathrm{M})$ \\
\hline dTMP & 40 & 10.60 & \\
\hline dUMP & 2100 & 3.50 & \\
\hline 5-Br-dUMP & 33 & 9.82 & \\
\hline 5-I-dUMP & 140 & 7.46 & \\
\hline 5-F-dUMP & 420 & 4.66 & \\
\hline AZTMP & & & 10 \\
\hline \multicolumn{4}{|c|}{ 5-methyl isodCMP } \\
\hline & & & 130 \\
\hline
\end{tabular}

Table 2. Kinetic parameters of base- and sugar-modified nucleotides for TMPKmt [12]. The affinity data were assessed at $340 \mathrm{~nm}$ via a spectrophotometric assay using coupling enzymes [27]. The concentration of ATP was kept constant at $0.5 \mathrm{mM}$.

\section{- Base modifications}

The 5-methyl group of dTMP was replaced by several halogen atoms [12]. The resulting analogs behave as substrates for TMPKmt, the best one being 5-Br-dUMP with a slight increase in affinity and negligible drop in velocity rate compared to dTMP (Table 2). Substitution by an iodine (5-I-dUMP) or a fluorine (5-F-dUMP) drastically decreased the affinity for the enzyme. X-ray crystallography showed that 5-I-dUMP binds to the phosphate acceptor binding site in a very similar fashion as dTMP [11]. Removal of the methyl group (dUMP) drastically affects the affinity (50-fold decrease) and the reaction rate (3-fold decrease). The 5halogenated deoxyuridines counterparts are competitive inhibitors of TMPKmt with affinities quite similar to their corresponding nucleoside monophosphates [28]. 5-Cl-dU and 5-Br-dU are remarkably specific for TMPKmt versus TMPKh. 


\section{- Sugar modification: AZTMP}

The 3'-hydroxyl group of dTMP, which adopts a C2'-endo conformation in the active site, participates in $\mathrm{Mg}^{2+}$ stabilization through interactions involving a water molecule and Asp9, the conserved acidic residue of the P-loop. Modifications of the 3'-position capable of destabilizing the interaction with the metal could form a firm basis to design compounds that specifically inhibit TMPKmt.

Substitution of the 3'-hydroxyl by an azido group affords AZTMP, which is a substrate for TMPKec [22] (with only a 2-fold reduction of $\mathrm{k}_{\text {cat }}$ ), TMPKy $[29,30]$, as well as TMPKh [21, 31], although with a low efficiency for the latter two enzymes. TMPKh is involved in the activation of the anti-HIV prodrug AZT. Since the enzyme poorly phosphorylates AZTMP, this step forms a bottleneck in the activation pathway that contributes to the toxicity and limited efficacy of AZT therapy [29, 32].

Interestingly, in the case of TMPKmt, the presence of an azido group completely suspends catalysis without changing the affinity. Thus, AZTMP behaves as a competitive inhibitor of TMPKmt with a $K_{i}$ value of $10 \mu M$, its nucleosidic counterpart AZT exhibiting a $\mathrm{K}_{\mathrm{i}}$ of $28 \mu \mathrm{M}[12,17]$. AZTMP probably inhibits TMPKmt by preventing $\mathrm{Mg}^{2+}$ from binding. Based on X-ray structures of TMPKmt in complex with dTMP and AZTMP, Fioravantini et al. rationalized this hypothesis as follows [33]. In a first step along the reaction pathway, dTMP interacts with D163 via an essential water molecule (Wat141). At this early stage of the reaction, the semi-open state of the enzyme prevents metal stabilization. In a second step, the LID region becomes organized as an $\alpha$-helix, thereby favoring the stabilization of D9, the conserved P-loop residue, and leading to metal binding. When AZTMP binds to the enzyme, the azido group takes the place of Wat141, which is prevented from binding and cannot stabilize D163. Consequently, LID closure, believed to be promoted by the anchoring of D163 on the nucleotide, and $\mathrm{Mg}^{2+}$ binding are not possible. Under these conditions, the only positively charged residue interacting with the acceptor phosphate is R95, which is insufficient to neutralize electrostatic repulsion between the anionic substrates dTMP and ATP. Additionally, the azido group establishes direct interactions with D9 before LID closure; therefore, D9, which also interacts with the $\alpha$-phophate group, is locked in a catalytically incompetent position.

\section{TMPKmt inhibitors closely related to thymidine}

Based on the X-ray structure of TMPKmt and the known affinities for the enzyme of a few compounds described above, it was decided to establish preliminary structure-activity relationships (SAR) by evaluating a 
series of nucleotides modified at the 2'- and 3'-positions or on the heterocyclic moiety of the dTMP-scaffold for their affinity toward TMPKmt. To address their selectivity, the most promising analogs were also evaluated for their affinity for TMPKh.

\section{- Base moiety modifications}

Because a 5-bromine (5Br-dUMP) is well accepted by the enzyme, analogs with sterically demanding substituents at the 5-position (e.g. a hydroxymethyl $\left(5 \mathrm{CH}_{2} \mathrm{OH}\right.$-dUMP), an ethyl and a bromovinyl group, or a furanyl, a benzyloxymethyl or a thienyl group, aimed at forming a stacking interaction with F36) were synthesized utilizing known synthetic transformations [34]. These inhibitors were less potent indicating that the cavity near the 5-position cannot be stretched too much. However, introduction of smaller 5-substituents (5fluorine, 5-hydroxyl and 5-trifluoromethyl) also imparted reduced activity. The binding of compound $5 \mathrm{CH}_{2} \mathrm{OH}$ dUMP to TMPKmt was further characterized by X-ray crystallography (Fig. 3). The structure verified our expectation by showing an anticipated hydrogen bond between $5 \mathrm{CH}_{2} \mathrm{OH}-\mathrm{dUMP}$ and a known water molecule (Wat12 in PDB entry 1G3U), as well as an unexpected binding mode with the hydroxyl group pointing toward the network of interactions between E6, R95, Y179, and S99. Both binding modes contributed to the rigidification of the dTMP binding pocket and explained why $5 \mathrm{CH}_{2} \mathrm{OH}$-dUMP is an inhibitor rather than a substrate, compared with 5-I-dUMP of comparable steric hindrance.
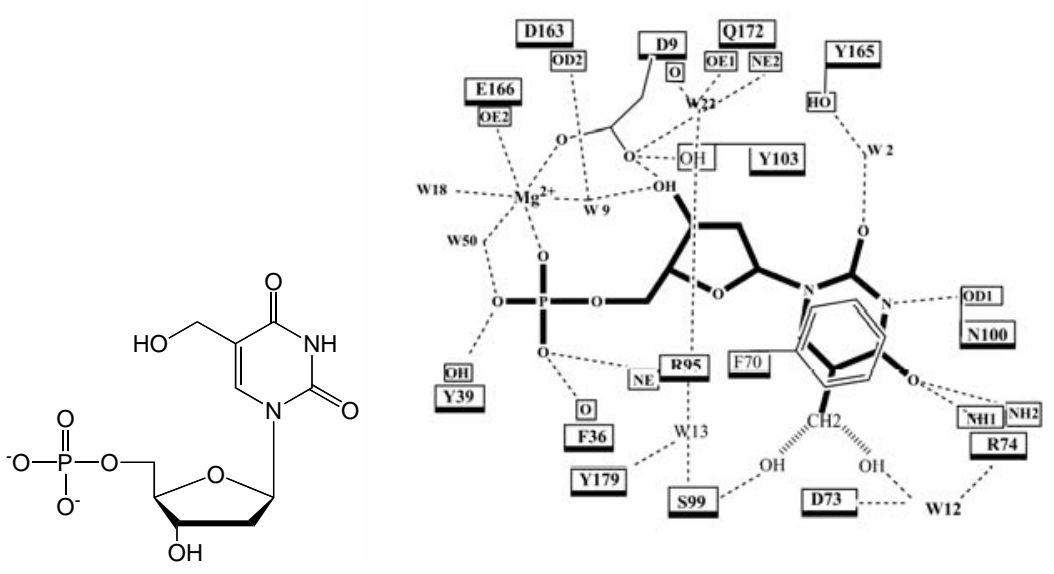

Fig. (3). Structure of the $5 \mathrm{CH}_{2} \mathrm{OH}-\mathrm{dUMP}$ (left) and the network of interactions of this inhibitor in the active site of TMPKmt (right).

Base-modified nucleosides (i.e. non-phosphorylated) were also explored: purine and pyrimidine nucleosides 
and C-nucleosides [28]. The best inhibitors remain the 5-halogenated derivatives of dU (isosteric and isoelectronic substituents of the methyl group) exhibiting high selectivity for TMPKmt compared to TMPKh.

\section{- 2'-Modifications}

Near the 2'-position lies Y103, believed to be responsible for discrimination between ribo- and deoxyribonucleotides. Consonantly, introduction of an $\alpha$-oriented 2'-hydroxyl group in dTMP negatively affected the affinity for TMPKmt, while the effect of a $\beta$-directed 2'-OH group was somewhat less detrimental. Interestingly, the same SAR was later observed upon introduction of a 2'-hydroxyl on the $\alpha$ - or $\beta$-face of AZT. dTMP decoration with an $\alpha$-directed 2'-amine group, envisioned to interact with Y103 via a cation-m interaction [35], also caused a drop in affinity. To sort out the influence of the size and electronegativity of 2'-substituents on the affinity for TMPKmt, $\alpha$-oriented fluorine and chlorine substituents were introduced in dTMP [36]. It could be expected that nucleotide analogs that exhibit an S-type conformation, similar to the one adopted by the furanose of dTMP in its complex to TMPKmt, would have a higher affinity than those that have to be forced into that configuration by the enzyme. Indeed the 2'-Cl dTMP exhibited appreciable affinity with a $\mathrm{K}_{\mathbf{i}}$ value of $19 \mu \mathrm{M}$. Modeling suggested that the introduction of the chlorine affects the position of the sugar ring in the active pocket. As a result the 2'-chlorine is believed to occupy the pocket where normally the 3'-hydroxyl group resides.

\section{- 3'-Modifications}

Due to the unique behavior of AZTMP towards TMPKmt in comparison to other TMPKs, 3'modifications in particular were considered to have potential for finding good and selective inhibitors. Because AZTMP interacts through its azido group with Asp9, we intended to establish an ionic interaction with Asp9 by introducing a 3'-amino group. However, upon reduction of AZTMP to the corresponding 3'-aminonucleotide, the $\mathrm{K}_{\mathbf{i}}$ value increased 20 -fold, indicating that the 3'-amino group does not interact with Asp9 as strongly as AZTMP. Remarkably, an analog with a 3'-fluorine behaved as a substrate for the enzyme rather than an inhibitor [36]. A series of nucleosides were then synthesized, that were based on the former SAR study and combined a 3'-azido or 3'-amino modification with a 2'-halogen substituent [37]. However, these analogs displayed only moderate binding affinity for TMPKmt. To explain this trend, their solution state conformational features were investigated using NMR spectroscopy and pseudorotational analysis. It was concluded that 
compounds of this series were strongly biased towards the Northern furanose ring conformation, whereas TMPKmt prefers the opposite Southern conformers.

Inspired by the fact that AZTMP perturbed the induced fit mechanism normally adopted by the enzyme and prevented the magnesium ion from binding in the active site, other modifications at C-3' that might produce similar effects, were considered. In the X-ray structure one could observe a cavity with the potential of accommodating larger substituents near the 3'-position. Hence, a series of 3'-C-branched-chain nucleotides were prepared (the 3'-C-hydroxymethyl (1), 3'-C-azidomethyl (2), 3'-C-aminomethyl (3) and 3'-C-fluoromethyl (4) derivatives of dTMP, scheme 1) [38]. These analogs were synthesized from a common key intermediate with judiciously chosen 5'- and 6'-O-protective groups (Scheme 1). 2',3'-Dideoxy-3'-branched chain analogs proved to be potent inhibitors of TMPKmt: within the series $3^{\prime}-\mathrm{CH}_{2} \mathrm{NH}_{2}$ (3), $3^{\prime}-\mathrm{CH}_{2} \mathrm{~N}_{3}$ (2) and $3^{\prime}-\mathrm{CH}_{2} \mathrm{~F}$ (4) nucleotides exhibited the highest affinities with $\mathrm{K}_{\mathrm{i}}$ values of $10.5,12$, and $15 \mu \mathrm{M}$, respectively. These results confirmed that TMPKmt tolerates the introduction of sterically demanding substituents at the 3'-position. Riboanalogs experienced a significant drop in affinity, probably due to steric hindrance of $\mathrm{Y} 103$. Although the 5'-Ophosphorylated compounds (1-4) had somewhat higher affinities for the enzyme, the parent nucleosides (5-8) generally exhibited affinities for TMPKmt in the same order of magnitude and displayed a superior selectivity profile versus TMPKh.
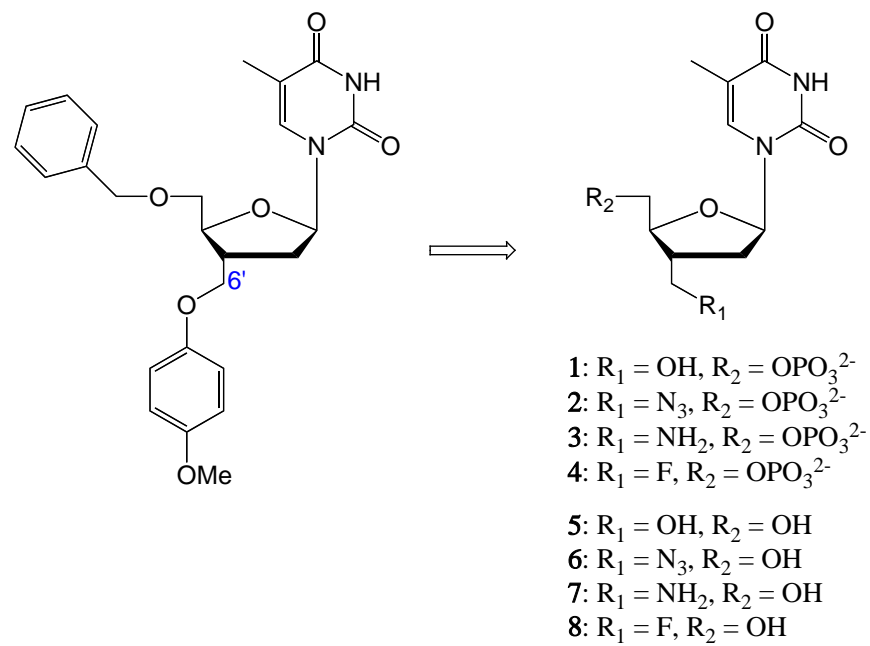

Scheme 1. Preparation of the 3'-branched chain nucleotides (1-4) and their nucleosidic counterparts (5-8) starting from a common key intermediate [38].

\section{- Computational procedures}

Based on a restricted set of 47 inhibitors of TMPKmt modified on the 5-, 2'-, 3'- and 5'-positions of dTMP (described above), the groups of Gopalakrishnan and Desijaru have developed models either to perform 
virtual screening [39] or 3D-QSAR analysis [40]. Both approaches take advantage of the solved 3D-structure of TMPKmt with dTMP [11]. In the first study, pharmacophore modeling and docking were carried out to define a composite screening model. Docking analysis confirmed the role of weak interactions in promoting enzyme selectivity toward deoxyribonucleotides. It also highlighted the importance of water-mediated cooperative networks and weak hydrogen bonds to ligand binding affinities. After its validation by screening a database of 2,000 molecules containing the training set of 47 molecules, the model was used for the virtual screening of 500,000 compounds. 186 molecules were further selected that could be categorized into five clusters [39]. Unfortunately, no binding data were reported. In the second study, different alignment methods were exploited to create molecular field analysis models. Again the same collection of 47 inhibitors of TMPKmt were selected either as the training or as the test set. One model out of three proved to be more predictive and was claimed informative about the chemical and structural features for TMPKmt inhibition [40].

\section{Drug design and identification of 'non nucleosidic' families}

With the aim to generate novel ligand families, a new fragment-based de novo drug design program was applied to the dTMP binding site of TMPKmt. This program called LEA3D combines 3D fragments and optimizes molecules by genetic algorithms [41]. It is associated to FlexX in order to evaluate the binding potency of the generated molecules. The active site of the enzyme was defined based on the coordinates of the 3D-structure of the dTMP/TMPKmt complex (1G3U) [11]. The thymine moiety was used as the first building block as most of the key interactions with dTMP are established with this part of the molecule. Moreover, very few modifications of the base moiety have led to potent ligands of TMPKmt. Different design cycles yielded a compound in which the sugar and the phosphate moieties of dTMP were replaced by a substituted benzyl group (Scheme 2, $\left.\mathrm{R}=-\left(\mathrm{CH}_{2}\right)_{2}-\mathrm{CONH}_{2}\right)$. A family of benzyl-thymine derivatives was derived from this compound and different members were synthesized [42, 43].
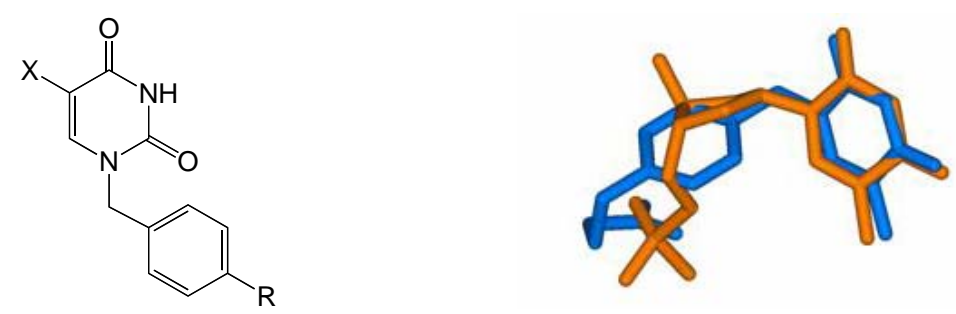

Scheme 2. Left panel: Benzyl-thymine derivatives. $X=\mathrm{CH}_{3}, \mathrm{Br}, \mathrm{Cl} ; \mathrm{R}=-\left(\mathrm{CH}_{2}\right)_{\mathrm{n}}-\mathrm{CONH}_{2},-\left(\mathrm{CH}_{2}\right)_{\mathrm{n}}-\mathrm{COOH}$, - 
$\left(\mathrm{CH}_{2}\right)_{n}-\mathrm{OH}$. Right panel: superposition of the crystallographic dTMP (in orange) with the predicted binding mode of one derivative $\left(X=\mathrm{CH}_{3}\right.$ and $\mathrm{R}=-\left(\mathrm{CH}_{2}\right)_{3}-\mathrm{COOH}$, in blue) using FlexX docking program (right) [41, 42].

The general synthetic route involved a palladium-catalyzed coupling reactions between an appropriate $p$-iodosubstituted benzyl pyrimidine and a suitably functionalized alkene or alkyne [42]. The arm length $(n=2-5)$, the saturation of the alkyl chain and the nature of the terminal function (amide, carboxylic acid or ester, alcohol) were varied. In the carboxylic series, the highest affinities ( $K_{i}$ between 6.5 and $\left.13 \mu \mathrm{M}\right)$ were obtained for the $C 4$ compounds $(n=3)$, whereas in the amide series a C6 linker $(n=5)$ was preferred. As previously noticed for nucleosidic inhibitors, the introduction of a bromine or a chloride at the 5-position improved the affinity of the benzyl derivatives. Some molecules showed moderate inhibitory potency on $M$. bovis (BCG strain) bacteria with $\mathrm{MIC}_{50}$ of $50 \mu \mathrm{g} / \mathrm{mL}$, whereas no cototoxicity was observed up to $400 \mu \mathrm{g} / \mathrm{mL}$ [42].

These results opened the way for a next generation of acyclic TMPKmt inhibitors (Scheme 3) that are accessible via a small number of synthetic steps [44].
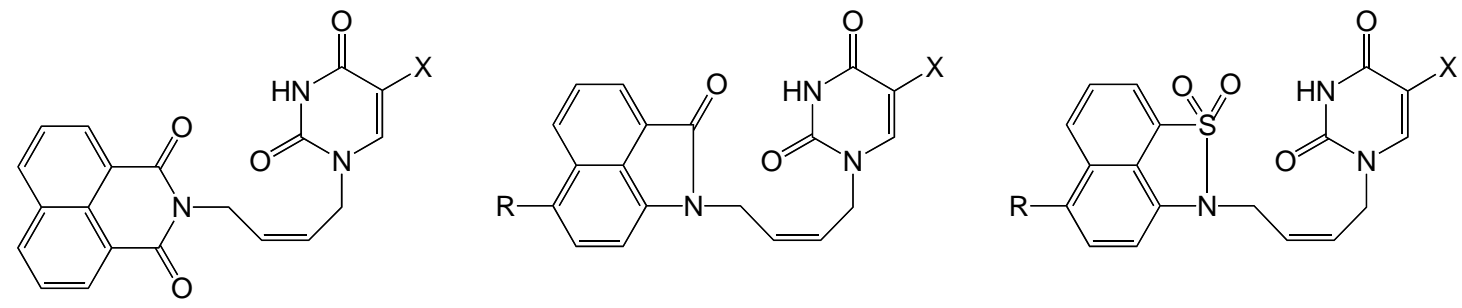

Scheme 3. Structures of the naphthalimide (left), naphtholactam (middle) and naphthosultam (right) series. $X=$ $\mathrm{CH}_{3}$ or $\mathrm{Br}[44]$.

Initially, a set of analogs with different spacers connecting a distal 1,8-naphthalimide to the thymine moiety was tested enabling to select (Z)-butenyl derivative as a privileged linker $\left(\mathrm{K}_{\mathrm{i}}=1.9\right.$ and $1.1 \mu \mathrm{M}$ for $\mathrm{X}=\mathrm{CH}_{3}$ and $\mathrm{Br}$ respectively; Scheme 3, left part). Keeping this optimized spacer, the naphtalimide moiety was modified or substituted for a naphtholactam or a naphthosultam moiety (Scheme 3). A naphtholactam or a naphthosultam proved superior and yielded inhibitors with affinities in the submicromolar range $\left(K_{i}=0.42\right.$ and $0.27 \mu \mathrm{M}$, respectively). Interestingly, these compounds were shown to be very selective for TMPKmt versus TMPKh, consistent with the absence of cytotoxicity on Vero cells up to $100 \mu \mathrm{g} / \mathrm{mL}$ [44]. Unfortunately none of these compounds were able to significantly inhibit the growth of mycobacteria, probably due to their very poor solubility in culture media. 


\section{Serendipitous discovery and SAR of bicyclic nucleosides}

In an effort to further optimize the inhibitory potency of the 3 '-C-branched-chain nucleosides, we envisoned to synthesize analogs of 7 with different substituents on the 3'- $\mathrm{CH}_{2}-\mathrm{NH}_{2}$ group. In an attempt to obtain a desired intermediate by simultaneous azide reduction and Barton deoxygenation at the 2'-position, some undesired compounds (Scheme 4) were obtained that, after deprotection, turned out to be potent and selective TMPKmt inhibitors ( $\mathrm{K}_{\mathrm{i}}$ of $3.5,13.5$ and $37 \mu \mathrm{M}$ for $\mathbf{9}, 10$ and 11 respectively) [45].

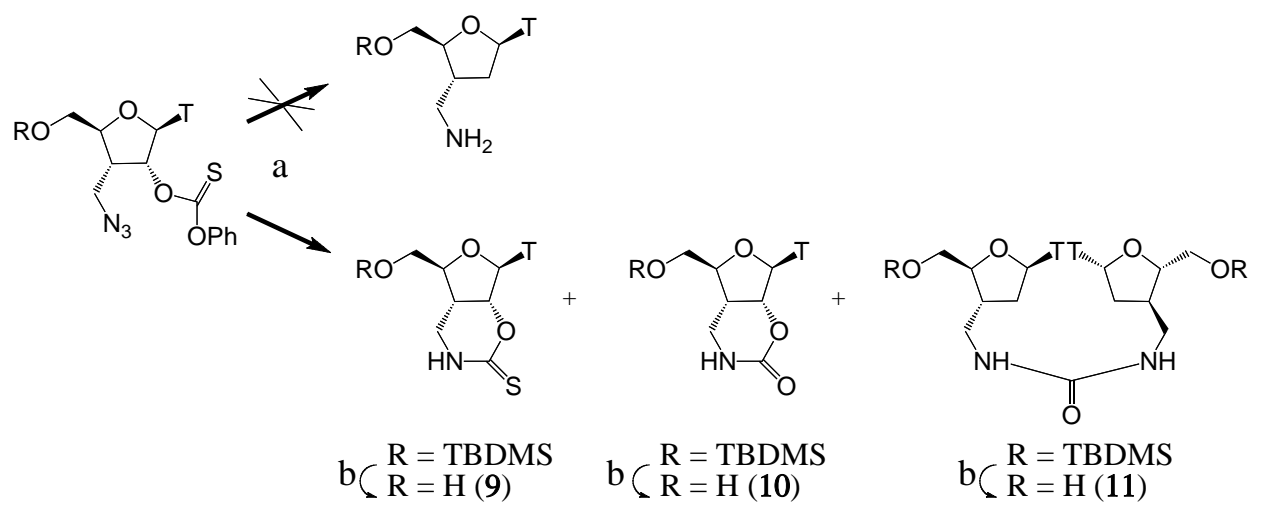

Scheme 4. Serendipitous discovery of TMPKmt inhibitors 9-11. Attempted simultaneous reduction of the azido function and Barton deoxygenation failed ( $\mathrm{a}, \mathrm{R}=\mathrm{TBDMS})$. Instead, three peculiar nucleoside analogs were formed, which proved active after removal of the silyl protective groups (b).

Compound 9 was modeled into the crystal structure of TMPKmt (Fig. 4). The six-membered ring, fused to the C-2', C-3'-bond of the sugar, apparently forces the 6'-nitrogen into the most appropriate position for interaction with D9. The sulphur atom experiences hydrophobic interactions with Y103 and Y165. When the sulphur atom in $\mathbf{9}$ is replaced by a smaller oxygen in 10, the cavity near the $3^{\prime}$-position is filled less efficiently, a result which is reflected in the somewhat lower affinity of $\mathbf{1 0 .}$ 


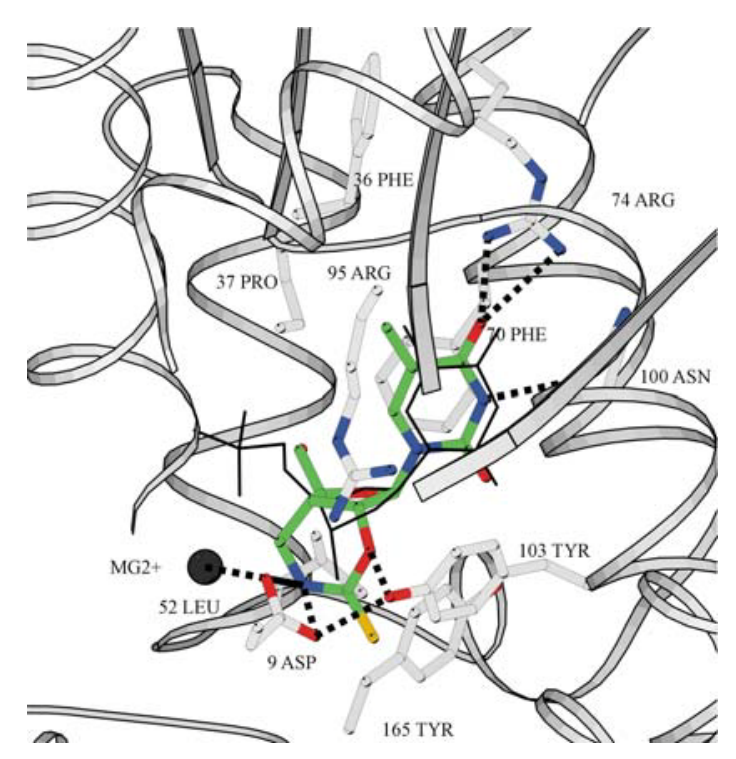

Fig. (4). Predicted binding mode of 9 with TMPKmt. A ribbon follows the backbone atoms of the enzyme. Hydrogen bonds are drawn as dashed lines. dTMP, as observed in the X-ray structure with TMPKmt, is shown in black sticks. Residues with atoms that make contact with $\mathbf{9}$, based on a Ligplot analysis, are F36, P37, L52, F70, R95, Y103 and Y165.

Since the bicyclic nucleoside 9 formed an interesting lead for finding inhibitors with an improved fit in the enzyme cavity, various analogs (Scheme 5) were synthesized [46].

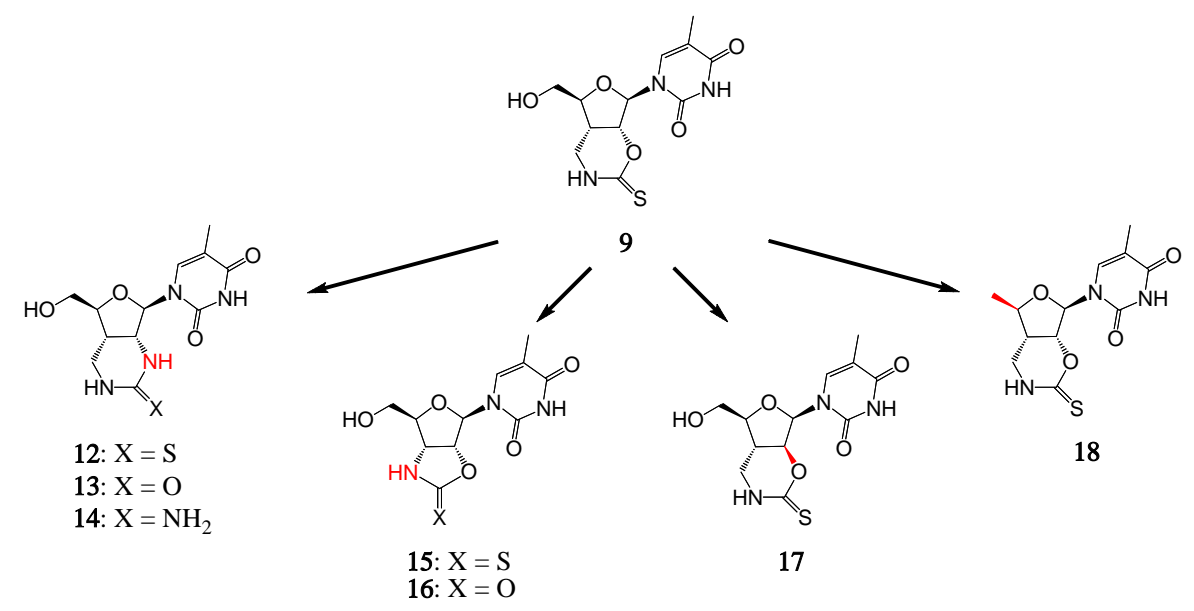

Scheme 5. Attempted optimization of the bicyclic nucleoside lead $\mathbf{9}$. Structural variations are highlighted in red.

In general, the accomplished modifications, such as replacement of the fused 6-membered thiocarbamate ring by a urea, thiourea or basic guanidine functionality $(13,12$ and 14$)$ or shrinking the 6 -membered to a 5 membered ring $(\mathbf{1 5}, \mathbf{1 6})$, led to a slight drop in affinity. A notable exception was the replacement of the 5'$\mathrm{CH}_{2} \mathrm{OH}$ group by a methyl, yielding a compound (18) that was almost twice as active $\left(\mathrm{K}_{\mathrm{i}}=2.3 \mu \mathrm{M}\right)$ as the 
original lead 9. Because this deoxygenated bicyclic nucleoside is supposed to exhibit superior physicochemical properties for penetrating the lipophilic cell wall of $M$. tuberculosis, its in vitro inhibitory activity against M. bovis BCG was investigated. It showed $100 \%$ inhibition of bacterial growth at $100 \mu \mathrm{g} / \mathrm{mL}$, while it did not demonstrate toxicity against VERO cell lines in a concentration range of $0-500 \mu \mathrm{g} / \mathrm{mL}$. As compound 9 failed to show any inhibition of bacterial growth at $64 \mu \mathrm{g} / \mathrm{mL}$, the activity of $\mathbf{1 8}$ represented significant progress.

\section{Optimization of the branched-chain nucleosides}

During the accidental formation of the bicyclic nucleosides, derivative 11, consisting of two 3'-branched thymidine units connected via a urea moiety, was also formed (Scheme 4). Due to the lack of a binding site for a second nucleoside at the $3^{\prime}$-position, the $K_{i}$ value of $11(37 \mu \mathrm{M})$ was most unexpected. A modeling experiment in GOLD showed that the sugar ring of the first monomer (I) binds in the dTMP pocket upside down, which enables hydrogen-bonding of its 5'-hydroxyl with D9 (Fig. 5).

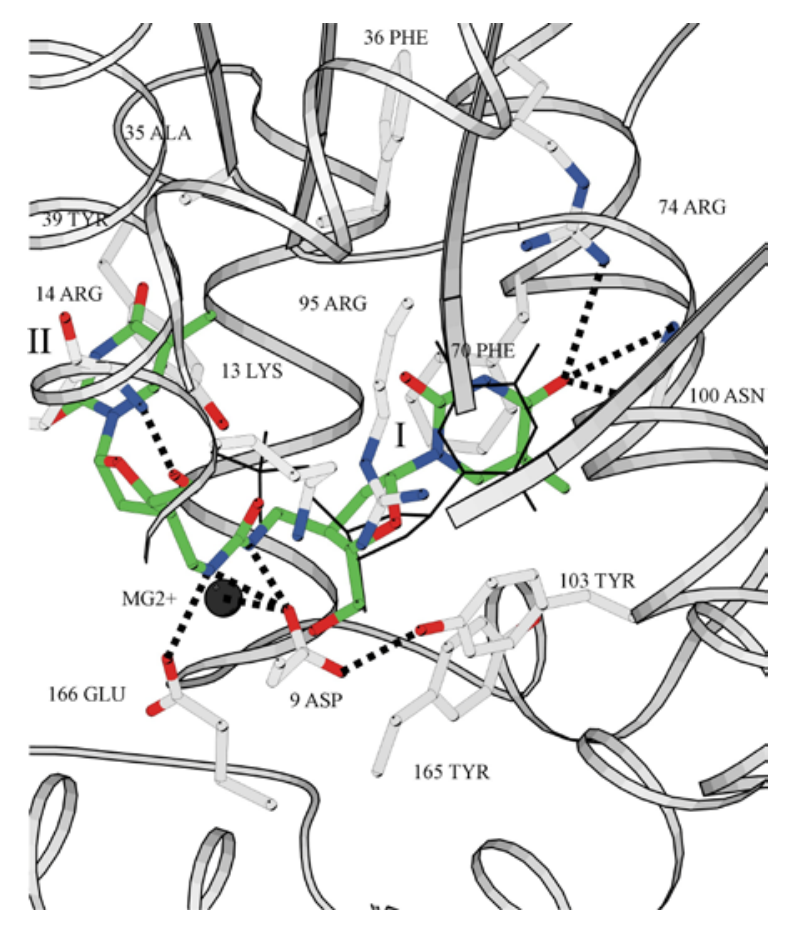

Fig. (5). Predicted binding mode with TMPKmt of $\mathbf{1 1}$ (color, I and II refer to the two monomers of 11) compared to the binding mode of dTMP (black sticks) as observed in the X-ray structure [11]. A ribbon follows the backbone atoms of the enzyme. Hydrogen bonds are drawn as dashed lines. Residues possessing atoms that make hydrophobic contact with 11, based on the Ligplot analysis, are K13, A35, Y39, F36, F70, R95, N100, Y103 and Y165. 
This binding mode permits the sugar ring of the second monomer (II) to be directed toward the outside of the enzyme, where normally the phosphoryl donor binds. Experiments performed with dTMP or ATP showed that this dinucleoside is a competitive inhibitor not only for dTMP but also for ATP ( $\mathrm{K}_{\mathrm{i}}=0.65 \mathrm{mM}$ at $\left.0.2 \mathrm{mM} \mathrm{dTMP}\right)$. These results are in agreement with the proposed binding mode. The low affinity of $\mathbf{1 1}$ for TMPKh (no inhibition at $1 \mathrm{mM}$ ) indicated that the flexibility towards the orientation of the sugar ring is specific for TMPKmt.

The fact that the two nucleosides in $\mathbf{1 1}$ were bound via their $\mathbf{3}^{\prime}$-positions made it a unique example of such a bisubstrate inhibitor, thereby paving the way for the design of much larger inhibitors of TMPKmt than explored so far. By synthesizing a series of substituted phenylthiourea derivatives of 3'- $\mathrm{CH}_{2} \mathrm{NH}_{2}-3^{\prime}-\mathrm{dT}$, the possibility to occupy the binding site which accommodated the second thymidine residue of $\mathbf{1 1}$ with different aryl moieties was further explored [47]. Based on the second Topliss method [48], a small group of phenyl isothiocyanates was selected as reagents for the final derivatisation step. The inhibitory potency order of the resulting thiourea could then be compared to the tabulated potency order calculated for various parameter dependencies relating to lipophilic, electronic and steric effects. After deduction of the probable operative parameters from the observed activity pattern, new isothiocyanates were selected (Scheme 6). This resulted in several analogs with excellent affinity for TMPKmt, e.g. 19 (7.2 $\mu \mathrm{M})$ and $20(5 \mu \mathrm{M})$.

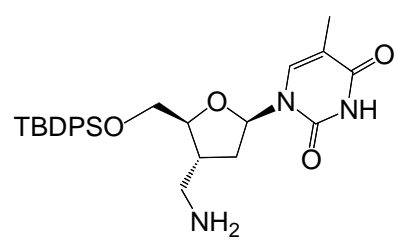

1. suitable isothiocyanate, DMF 2. TBAF, THF

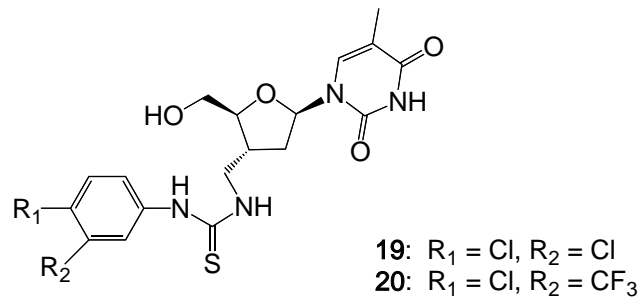

Scheme 6. Synthesis of 3'-C-branched $\beta$-thioureaderivatives [47].

In a next step it was decided to explore if an alternative sugar scaffold could be used to impose the favorable relative orientation of the thymine and the phenylthiourea moieties for TMPKmt inhibition [47]. It was hypothesized that an $\alpha$-nucleoside in which the 5'-position served as the thiourea anchor might show potential towards this end. This hypothesis was supported by the discovery that $\alpha$-thymidine 5 '-monophosphate ( $\alpha$ dTMP) was accepted by TMPKmt as a substrate $\left(K_{m}=15 \mu \mathrm{M}\right)$, indicating that inversion of configuration at the anomeric position does not prevent the $5^{\prime}-\mathrm{OP}(\mathrm{O})(\mathrm{OH})_{2}$ moiety from being favorably oriented in order to operate as a phosphate acceptor. From a small library of 5'- $\mathrm{N}$-arylthiourea derivatives, 22 (Fig. 6) emerged as one of the most potent TMPKmt inhibitors to date $\left(K_{i}=0.6 \mu M\right)$ [47]. Since it can be prepared in a limited number of 
steps from the easily available $\alpha$-thymidine, this compound represents a conceivably more attractive lead than the branched-chain nucleosides 19 and 20. Among the series of 5'-N-arylthiourea derivatives including $\mathbf{2 2 ,}$ seven showed 40 to $50 \%$ inhibition of $M$. tuberculosis growth at $6 \mu \mathrm{g} / \mathrm{mL}$, while demonstrating virtually no toxicity against VERO cell lines at a concentration up to $500 \mu \mathrm{g} / \mathrm{mL}$ [47]. Based on the observation that the 3'deoxy and the 3'-deoxy-2',3'-didehydro analogs of 22 exhibit slightly higher $\mathrm{K}_{i}$ values (2.3 and $\left.3.8 \mu \mathrm{M}\right)$, the 3'$\mathrm{OH}$ group is assumed to contribute to the overall affinity. Interestingly, the azido and amino precursors also caused TMPKmt inhibition with $\mathrm{K}_{\mathrm{i}}$ values of 26 and $16 \mu \mathrm{M}$. These results can be rationalized by superimposing 20 with the enzyme-bound conformation of 3'-branched chain nucleoside 7 (Fig. 6). Indeed, upon superposition of the thymine moieties, the 4'-aminomethyl moiety of $\mathbf{2 1}$ occupies the same space as the 3'-aminomethyl moiety of 7 .
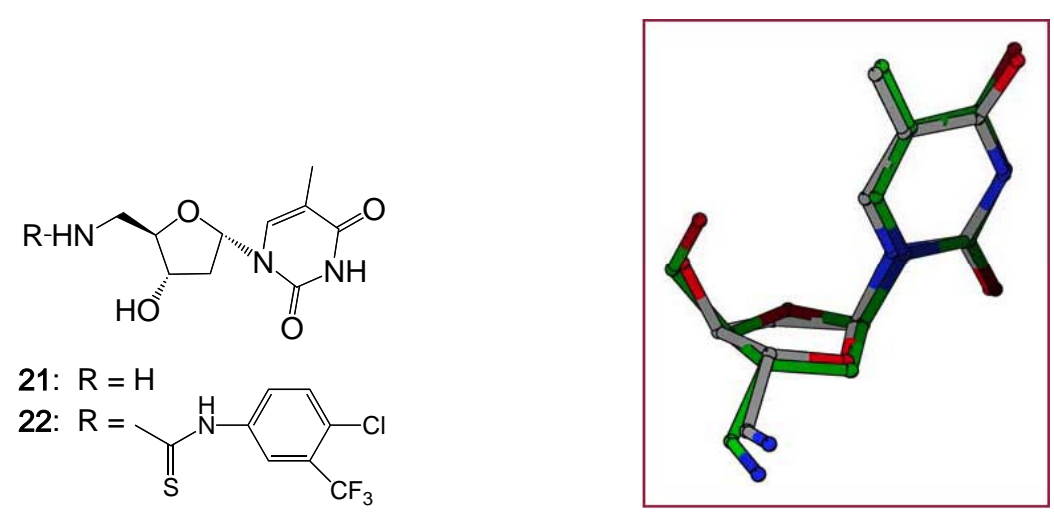

Fig. (6). Left panel: 5'-substituted $\alpha$-thymidine derivatives. Right panel: superimposition of 21 (grey) with 7 (green) in the presumed enzyme-bound conformation. Note the similar orientation of the amino groups (in blue).

A 3D pharmacophore mapping was performed by the groups of Andrade and Hopfinger based upon the application of receptor-independent $(R I)$ 4D-QSAR formalism to the series of 5'-thiourea-substituted $\alpha$ thymidine derivatives. A set of 34 compounds including the dinucleoside 11 [47] were selected to develop 4DQSAR models [49]. Although the crystal structure of TMPKmt is available [11], the 3D structure of the receptor was neglected because of uncertainty in the binding mode of the ligands. One model out of ten was further considered and was shown to be $75 \%$ predictive on a test set of four compounds. Based on this model, Andrade et al. proposed regions to be further explored such as the 5'-arylthiourea moiety, such as the sugarpyrimidine ring structure and the region of the 5 -arylthiourea moiety. Moreover, this study highlighted the importance of lipophilic substituents on the 5 -aryl moiety that possibly represent an additional pharmacophore 
site responsible for the higher inhibition potency of these derivatives [49]. For a better validation of the model, this study has been expanded to a larger number of inhibitors of TMPKmt with more chemical diversity. Moreover, the autors will compare different subclasses of analog inhibitors, to map out subtle differences in binding pharmacophores for high potency inhibitors [50].

\section{CONCLUSION}

The search for new antituberculosis drugs has rapidly expanded in the late 1990s thanks to the complete sequencing of the M. tuberculosis genome. The concomitant development of molecular genetics of $M$. tuberculosis and other mycobacteria has greatly facilitated target identification and validation. In this context, TMPKmt was chosen as a key enzyme in the metabolism of nucleotides. Even if TMPK is an ubiquitous enzyme, peculiar properties of TMPKmt came to light by biochemical and structural studies. A de novo design approach has afforded promising TMPKmt inhibitors with the required selectivity vis-à-vis the human enzyme, consistent with the absence of cytotoxicity of these compounds. The available X-ray structures proved a valuable tool to guide modifications of first series of nucleosidic compounds and to identify new families of inhibitors by in silico approaches. Different families of nucleosidic and non nucleosidic compounds were reported with $\mathrm{K}_{\mathrm{i}}$ values in the micromolar and submicromolar range. Various analogues were able to inhibit mycobacterial growth, thereby validating TMPKmt as a new target for the search of new antituberculosis agents. However, up to now the MIC on mycobacterial cultures remains above the micromolar range (10 $\mu \mathrm{M})$, probably due to uptake limitations. However, recent reports on potent and selective antimycobacterial activities for pyrimidine dideoxynucleosides featuring 5-alkynyl substituents [51] and nucleoside bisubstrate analogs [52] are encouraging and seem to suggest that further finetuning of the physicochemical properties may one day afford TMPK inhibitors with the expected antimycobacterial activity.

\section{ACKOWLEDGEMENTS}

S.V.C. thanks the UGent Research Fund (BOF, Ghent University), the Fund for Scientific Research-Flanders (F.W.O.-Vlaanderen) and the Institute for the Promotion of Innovation by Science and Technology in Flanders (IWT) for scholarships and funding. S.P and H. M.-L. gratefully acknowledge the Institut Pasteur (GPH Tuberculose, DVPI), CNRS, INSERM, and Ministère de la Recherche (ACI). 


\section{REFERENCES}

1. Global tuberculosis control : epidemiology, strategy, financing : WHO report 2009., WHO Press.

2. Dorman, S. E. \& Chaisson, R. E. (2007) From magic bullets back to the magic mountain: the rise of extensively drug-resistant tuberculosis, Nat Med. 13, 295-8.

3. Aagaard, C., Dietrich, J., Doherty, M. \& Andersen, P. (2009) TB vaccines: current status and future perspectives, Immunol Cell Biol. 87, 279-86.

4. Janin, Y. L. (2007) Antituberculosis drugs: ten years of research, Bioorg Med Chem. 15, 2479-513.

5. Sacchettini, J. C., Rubin, E. J. \& Freundlich, J. S. (2008) Drugs versus bugs: in pursuit of the persistent predator Mycobacterium tuberculosis, Nat Rev Microbiol. 6, 41-52.

6. Cole, S. T., Brosch, R., Parkhill, J., Garnier, T., Churcher, C., Harris, D., Gordon, S. V., Eiglmeier, K., Gas, S., Barry, C. E., 3rd, Tekaia, F., Badcock, K., Basham, D., Brown, D., Chillingworth, T., Connor, R., Davies, R., Devlin, K., Feltwell, T., Gentles, S., Hamlin, N., Holroyd, S., Hornsby, T., Jagels, K. \& Barrell, B. G. (1998) Deciphering the biology of Mycobacterium tuberculosis from the complete genome sequence., Nature. 393, 537-44.

7. Sassetti, C. M., Boyd, D. H. \& Rubin, E. J. (2003) Genes required for mycobacterial growth defined by high density mutagenesis, Mol Microbiol. 48, 77-84.

8. Zhang, Y., Post-Martens, K. \& Denkin, S. (2006) New drug candidates and therapeutic targets for tuberculosis therapy, Drug Discov Today. 11, 21-7.

9. Yan, H. \& Tsai, M.-D. (1999) Nucleoside monophosphate kinases: Structure, mechanism, and substrate specificity, Adv. Enzymol. Related Areas Molec. Biol. 73, 103-133.

10. Jong, A. Y., Kuo, C.-L. \& Campbell, J. L. (1984) The CDC8 gene of yeast encodes thymidylate kinase, J. Biol. Chem. 259, 11052-11059.

11. Li de la Sierra, I., Munier-Lehmann, H., Gilles, A. M., Barzu, O. \& Delarue, M. (2001) X-ray structure of TMP kinase from Mycobacterium tuberculosis complexed with TMP at $1.95 \AA$ resolution, Journal of Molecular Biology. 311, 87-100.

12. Munier-Lehmann, H., Chaffotte, A., Pochet, S. \& Labesse, G. (2001) Thymidylate kinase of Mycobacterium tuberculosis: A chimera sharing properties common to eukaryotic and bacterial enzymes, Protein Sci. 10, 11951205.

13. Brosch, R., Gordon, S. V., Garnier, T., Eiglmeier, K., Frigui, W., Valenti, P., Dos Santos, S., Duthoy, S., Lacroix, C., Garcia-Pelayo, C., Inwald, J. K., Golby, P., Garcia, J. N., Hewinson, R. G., Behr, M. A., Quail, M. A., Churcher, C., Barrell, B. G., Parkhill, J. \& Cole, S. T. (2007) Genome plasticity of BCG and impact on vaccine efficacy, Proc Natl Acad Sci U S A. 104, 5596-601.

14. Cole, S. T., Eiglmeier, K., Parkhill, J., James, K. D., Thomson, N. R., Wheeler, P. R., Honore, N., Garnier, T., Churcher, C., Harris, D., Mungall, K., Basham, D., Brown, D., Chillingworth, T., Connor, R., Davies, R. M., Devlin, K., Duthoy, S., Feltwell, T., Fraser, A., Hamlin, N., Holroyd, S., Hornsby, T., Jagels, K., Lacroix, C., Maclean, J., Moule, S., Murphy, L., Oliver, K., Quail, M. A., Rajandream, M. A., Rutherford, K. M., Rutter, S., Seeger, K., Simon, S., Simmonds, M., Skelton, J., Squares, R., Squares, S., Stevens, K., Taylor, K., Whitehead, S., Woodward, J. R. \& Barrell, B. G. (2001) Massive gene decay in the leprosy bacillus, Nature. 409, 1007-11.

15. Garnier, T., Eiglmeier, K., Camus, J. C., Medina, N., Mansoor, H., Pryor, M., Duthoy, S., Grondin, S., Lacroix, C., Monsempe, C., Simon, S., Harris, B., Atkin, R., Doggett, J., Mayes, R., Keating, L., Wheeler, P. R., Parkhill, J., Barrell, B. G., Cole, S. T., Gordon, S. V. \& Hewinson, R. G. (2003) The complete genome sequence 
of Mycobacterium bovis, Proc Natl Acad Sci U S A. 100, 7877-82.

16. Saito, H. \& Tomioka, H. (1984) Thymidine kinase of bacteria: Activity of the enzyme in actinomycetes and related organisms, J. Gen. Microbiol. 130, 1863-1870.

17. Pochet, S., Dugue, L., Douguet, D., Labesse, G. \& Munier-Lehmann, H. (2002) Nucleoside analogues as inhibitors of thymidylate kinases: possible therapeutic applications, ChemBioChem. 3, 108-10.

18. Kandeel, M. \& Kitade, Y. (2008) Molecular characterization, heterologous expression and kinetic analysis of recombinant Plasmodium falciparum thymidylate kinase, J Biochem. 144, 245-50.

19. Lavie, A., Konrad, M., Brundiers, R., Goody, R. S., Schlichting, I. \& Reinstein, J. (1998) Crystal structure of yeast thymidylate kinase complexed with the bisubstrate inhibitor P1-(5'-adenosyl) P5-(5'-thymidyl) pentaphosphate (TP5A) at $2.0 \AA$ resolution: implications for catalysis and AZT activation, Biochemistry. 37, 3677-86.

20. Saraste, M., Sibbald, P. R. \& Wittinghofer, A. (1990) The P-loop-a common motif in ATP- and GTPbinding proteins, Trends Biochem. Sci. 15, 430-434.

21. Brundiers, R., Lavie, A., Veit, T., Reinstein, J., Schlichting, I., Ostermann, N., Goody, R. S. \& Konrad, M. (1999) Modifying human thymidylate kinase to potentiate azidothymidine activation, J Biol Chem. 274, 3528992.

22. Lavie, A., Ostermann, N., Brundiers, R., Goody, R. S., Reinstein, J., Konrad, M. \& Schlichting, I. (1998) Structural basis for efficient phosphorylation of 3 -azidothymidine monophosphate by Escherichia coli thymidylate kinase, Proc. Natl. Acad. Sci. USA. 95, 14045-14050.

23. Fioravantia, E., Haouz, A., Ursby, T., Munier-Lehmann, H., Delarue, M. \& Bourgeois, D. (2003) Mycobacterium tuberculosis Thymidylate Kinase: Structural Studies of Intermediates along the Reaction Pathway, J. Mol. Biol. 327, 1077-1092.

24. Matte, A., Tari, L. W. \& Delbaere, L. T. (1998) How do kinases transfer phosphoryl groups?, Structure. 6 , 413-9.

25. Vonrhein, C., Schlauderer, G. J. \& Schulz, G. E. (1995) Movie of the structural changes during a catalytic cycle of nucleoside monophosphate kinases, Structure. 3, 483-490.

26. Rhoads, D. G. \& Lowenstein, J. M. (1968) Initial velocity and equilibrium kinetics of myokinase, J. Biol. Chem. 243, 3963-3972.

27. Blondin, C., Serina, L., Wiesmüller, L., Gilles, A.-M. \& Bârzu, O. (1994) Improved spectrophotometric assay of nucleoside monophosphate kinase activity using the pyruvate kinase/lactate dehydrogenase coupling system, Anal. Biochem. 220, 219-221.

28. Pochet, S., Dugué, L., Labesse, G., Delepierre, M. \& Munier-Lehmann, H. (2003) Comparative study of purine and pyrimidine nucleoside analogues acting on the thymidylate kinases of Mycobacterium tuberculosis and of humans, ChemBioChem. 4, 742-747.

29. Lavie, A., Schlichting, I., Vetter, I. R., Konrad, M., Reinstein, J. \& Goody, R. S. (1997) The bottleneck in AZT activation, Nature Med. 3, 922-924.

30. Lavie, A., Vetter, I. R., Konrad, M., Goody, R. S., Reinstein, J. \& Schlichting, I. (1997) Structure of thymidylate kinase reveals the cause behind the limiting step in AZT activation, Nat. Struct. Biol. 4, 601-604.

31. Ostermann, N., Lavie, A., Padiyar, S., Brundiers, R., Veit, T., Reinstein, J., Goody, R. S., Konrad, M. \& Schlichting, I. (2000) Potentiating AZT activation: structures of wild-type and mutant human thymidylate kinase suggest reasons for the mutants' improved kinetics with the HIV prodrug metabolite AZTMP, J Mol Biol. 304, 43-53. 
32. Lavie, A. \& Konrad, M. (2004) Structural requirements for efficient phosphorylation of nucleotide analogs by human thymidylate kinase, Mini Rev Med Chem. 4, 351-9.

33. Fioravanti, E., Adam, V., Munier-Lehmann, H. \& Bourgeois, D. (2005) The crystal structure of Mycobacterium tuberculosis thymidylate kinase in complex with 3'-azido-deoxythymidine-monophosphate (AZTMP) suggests a mechanism for competitive inhibition, Biochemistry. 44, 130-7.

34. Haouz, A., Vanheusden, V., Munier-Lehmann, H., Froeyen, M., Herdewijn, P., Van Calenbergh, S. \& Delarue, M. (2003) Enzymatic and structural analysis of inhibitors designed against M. tuberculosis thymidylate kinase: new insights into the phosphoryl transfer mechanism, J. Biol. Chem. 278, 4963-4971.

35. Mo, Y., Subramanian, G., Gao, J. \& Ferguson, D. M. (2002) Cation-ா Interactions: An Energy Decomposition Analysis and Its Implication in $\delta$-Opioid Receptor-Ligand Binding, Journal of the American Chemical Society. 124, 4832-37.

36. Vanheusden, V., Munier-Lehmann, H., Pochet, S., Herdewijn, P. \& Van Calenbergh, S. (2002) Synthesis and evaluation of thymidine-5'-O-monophosphate analogues as inhibitors of Mycobacterium tuberculosis thymidylate kinase, Bioorganic \& Medicinal Chemistry Letters. 12, 2695-8.

37. Van Rompaey, P., Nauwelaerts, K., Vanheusden, V., Rozenski, J., Munier-Lehmann, H., Herdewijn, P. \& Van Calenbergh, S. (2003) Mycobacterium tuberculosis Thymidine Monophosphate Kinase Inhibitors: Biological Evaluation and Conformational Analysis of 2'- and 3'-Modified Thymidine Analogues, European Journal of Organic Chemistry, 2911-2918.

38. Vanheusden, V., Munier-Lehmann, H., Froeyen, M., Dugué, L., Heyerick, A., De Keukeleire, D., Pochet, S., Busson, R., Herdewijn, P. \& Van Calenbergh, S. (2003) 3'-C-branched-chain-substituted nucleosides and nucleotides as potent inhibitors of Mycobacterium tuberculosis thymidine monophosphate kinase., J. Med. Chem. 46, 3811-21.

39. Gopalakrishnan, B., Aparna, V., Jeevan, J., Ravi, M. \& Desiraju, G. R. (2005) A virtual screening approach for thymidine monophosphate kinase inhibitors as antitubercular agents based on docking and pharmacophore models, J Chem Inf Model. 45, 1101-8.

40. Aparna, V., Jeevan, J., Ravi, M., Desiraju, G. R. \& Gopalakrishnan, B. (2006) 3D-QSAR studies on antitubercular thymidine monophosphate kinase inhibitors based on different alignment methods, Bioorg Med Chem Lett. 16, 1014-20.

41. Douguet, D., Munier-Lehmann, H., Labesse, G. \& Pochet, S. (2005) LEA3D : a computer-aided ligand design for structure-based drug design, J. Med. Chem. 48, 2457-68.

42. Gasse, C., Douguet, D., Huteau, V., Marchal, G., Munier-Lehmann, H. \& Pochet, S. (2008) Substituted benzyl-pyrimidines targeting thymidine monophosphate kinase of Mycobacterium tuberculosis: Synthesis and in vitro anti-mycobacterial activity, Bioorg Med Chem. 16, 6075-85.

43. Munier-Lehmann, H., Douguet, D., Labesse, G. \& Pochet, S. (2004) Aryl pyrimidyl compounds, pharmaceutical compositions comprising them, their use as antimicrobial agents in

44. Familiar, O., Munier-Lehmann, H., Negri, A., Gago, F., Douguet, D., Rigouts, L., Hernandez, A. I., Camarasa, M. J. \& Perez-Perez, M. J. (2008) Exploring Acyclic Nucleoside Analogues as Inhibitors of Mycobacterium tuberculosis Thymidylate Kinase, ChemMedChem.

45. Vanheusden, V., Munier-Lehmann, H., Froeyen, M., Busson, R., Rozenski, J., Herdewijn, P. \& Van Calenbergh, S. (2004) Discovery of bicyclic thymidine analogues as selective and high-affinity inhibitors of Mycobacterium tuberculosis thymidine monophosphate kinase, J. Med. Chem. 47, 6187-94.

46. Van Daele, I., Munier-Lehmann, H., Hendrickx, P. M., Marchal, G., Chavarot, P., Froeyen, M., Qing, L., 
Martins, J. C. \& Van Calenbergh, S. (2006) Synthesis and Biological Evaluation of Bicyclic Nucleosides as Inhibitors of M. tuberculosis Thymidylate Kinase, ChemMedChem. 1, 1081-90.

47. Van Daele, I., Munier-Lehmann, H., Froeyen, M., Balzarini, J. \& Calenbergh, S. V. (2007) Rational Design of $5^{\prime}$-Thiourea-Substituted alpha-Thymidine Analogues as Thymidine Monophosphate Kinase Inhibitors Capable of Inhibiting Mycobacterial Growth, J Med Chem. 50, 5281-5292.

48. Topliss, J. G. (1977) A manual method for applying the Hansch approach to drug design, J Med Chem. 20 , 463-9.

49. Andrade, C. H., Pasqualoto, K. F., Ferreira, E. I. \& Hopfinger, A. J. (2009) Rational design and 3Dpharmacophore mapping of 5 '-thiourea-substituted alpha-thymidine analogues as mycobacterial TMPK inhibitors, J Chem Inf Model. 49, 1070-8.

50. Andrade, C.H., personal communication.

51. Rai, D., Johar, M., Srivastav, N. C., Manning, T., Agrawal, B., Kunimoto, D. Y. \& Kumar, R. (2007) Inhibition of Mycobacterium tuberculosis, Mycobacterium bovis, and Mycobacterium avium by novel dideoxy nucleosides, J Med Chem. 50, 4766-74.

52. Neres, J., Labello, N. P., Somu, R. V., Boshoff, H. I., Wilson, D. J., Vannada, J., Chen, L., Barry, C. E., 3rd, Bennett, E. M. \& Aldrich, C. C. (2008) Inhibition of siderophore biosynthesis in Mycobacterium tuberculosis with nucleoside bisubstrate analogues: structure-activity relationships of the nucleobase domain of 5'-O-[N(salicyl)sulfamoyl]adenosine, J Med Chem. 51, 5349-70. 